\title{
Photogrammetry for accurate model deformation measurement in a supersonic wind tunnel
}

\author{
Michela Gramola $^{1} \odot$. Paul J. K. Bruce ${ }^{1} \cdot$ Matthew Santer $^{1}$
}

Received: 15 September 2018 / Revised: 14 November 2018 / Accepted: 15 November 2018 / Published online: 3 December 2018

(c) The Author(s) 2018

\begin{abstract}
The interest in adaptive devices for high-speed applications leads to the need for an accurate and reliable technique to obtain model deformation measurements during experiments. Point-tracking photogrammetry has been applied to supersonic wind tunnel testing, using four Phantom high-speed cameras placed on either side of the working section, where coded targets were applied to the surface of interest. Calibration experiments on a solid plate beneath a $M=1.4$ normal shock and a $M=2$ oblique shock allowed the quantification of the sources of optical distortion, namely the wind tunnel glass windows and aerodynamic effects (the lower pressure in the working section and the interaction between shock waves and the boundary layer). A correction matrix was applied to account for the optical distortion due to the glass, and the root-mean-square error due to aerodynamic effects $(<0.03 \mathrm{~mm})$ is believed to be negligible for applications with significant displacements (of the order of $1 \mathrm{~mm}$ ). The application of photogrammetry to a flexible shock control bump has shown that the bump shape can be detected accurately, while disclosing some complex 3D effects that could not have been revealed by spanwise-averaged techniques such as schlieren photography.
\end{abstract}

Graphical abstract 3D reconstruction of an adaptive shock control bump from photogrammetry with the corresponding shadowgraph picture showing the $\lambda$-shock structure (dimensions in $\mathrm{mm}$ )

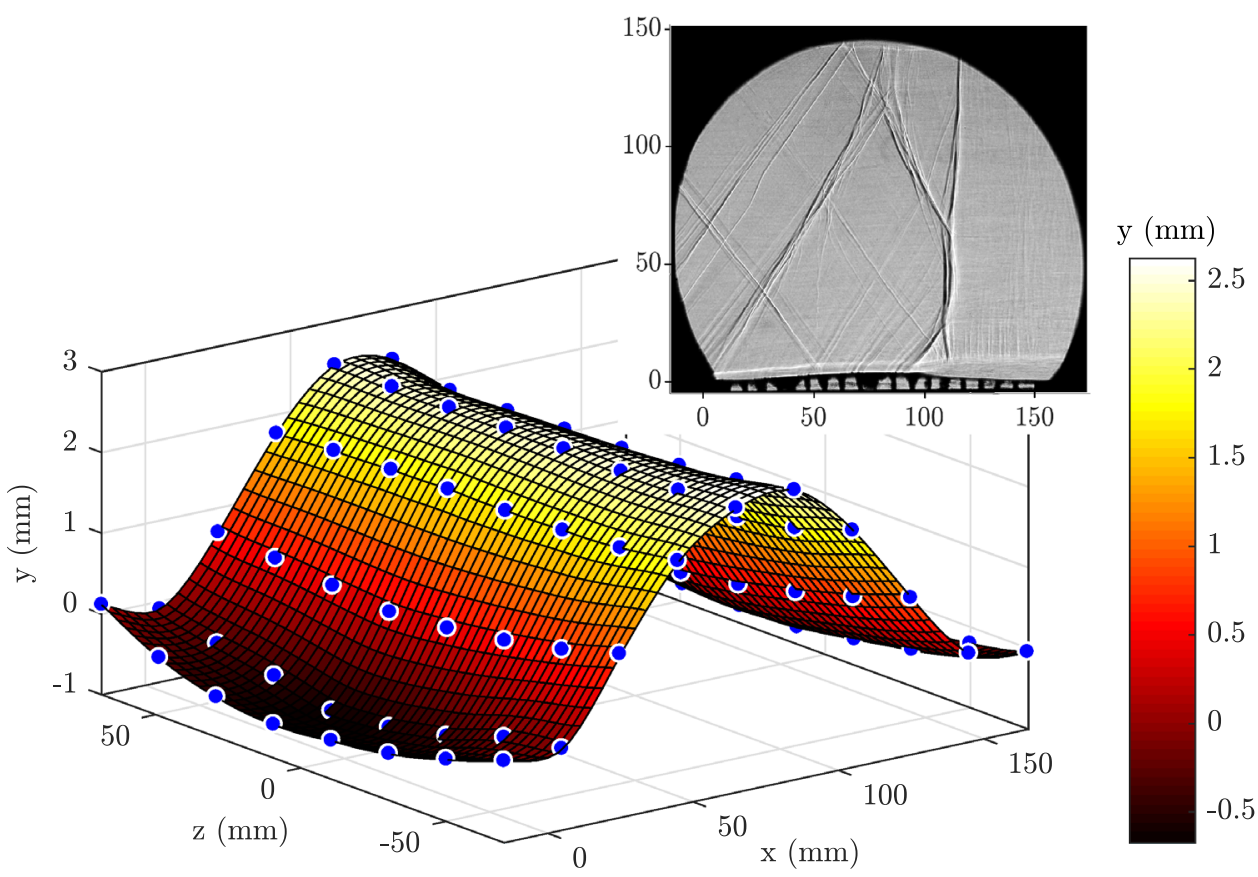

Extended author information available on the last page of the article 


\section{Introduction}

Given the interest in adaptive devices for high-speed applications, efforts have been focused on understanding their complex aerostructural behavior. Experiments in supersonic wind tunnels investigate the aerodynamic aspects, and, to fully understand the aeroelastic properties of a model and validate simulations, it is essential to obtain accurate deformation data. For 2D models, deformations are often estimated from schlieren videos, generating a spanwise projected average and assuming that displacements will also be 2D (Gramola et al. 2018). However, the flow in a supersonic wind tunnel is often highly $3 \mathrm{D}$ (Grossman and Bruce 2018), meaning that even 2D models may exhibit some 3D deformations, and high accuracy will not be achieved with projected averages. Furthermore, large density gradients in regions of the flow containing compressible boundary layers and shock waves will lead to additional errors in schlieren measurements, caused by optical distortion.

Photogrammetry is an optical technique that was originally developed for topographical applications to map points from $2 \mathrm{D}$ image coordinates to $3 \mathrm{D}$ spatial coordinates. In the past decades, it has received considerable attention as a noncontact measurement technique for aerospace research; the most recent approaches and applications being discussed by Baqersad et al. (2017). Based on the type of targets used for tracking, they distinguish between a point-tracking approach (which detects the displacement of optical targets mounted to the structure), digital image correlation (based on applying a high contrast speckle pattern to the surface of interest to measure full-field displacements and strains), and a target-less approach (which relies on the motion of internal features or edges of a structure). This paper will focus on point-tracking photogrammetry.

Photogrammetry has proven to provide sufficient accuracy and resolution at a relatively low cost, making it suitable for aeroelastic research, with applications including wind tunnel experiments (Graves et al. 2003; Spain et al. 2004; Barrows 2007; Zhang et al. 2012; Quix et al. 2015; Schairer et al. 2017, 2018), and ground (Kirmse et al. 2016) and flight testing (Burner et al. 2003), using different approaches in terms of target choice, object illumination, camera location, and post-processing algorithms. Photogrammetry has been used in experiments in subsonic, transonic, supersonic, and hypersonic wind tunnels of very different sizes, with test section cross-sectional areas from the order of $0.01 \mathrm{~m}^{2}$ up to nearly $900 \mathrm{~m}^{2}$ (Schairer et al. 2018). In recent years, photogrammetry has been increasingly applied to study transient phenomena (Graves et al. 2003; Schairer et al. 2018) and the dynamics of vibrating structures (Castellini et al. 2017; Wang et al. 2018).
Given that photogrammetry is an optical technique applied in complex aerodynamic conditions, uncertainty estimation is critical and extremely sensitive to the experimental setup. Kirmse et al. (2010) investigated the impact of strong density gradients on the accuracy of model deformation measurements by means of the Image Pattern Correlation Technique (IPCT). Experiments were performed on a 2D-NACA0010 airfoil at $M_{\infty}=0.8$ and $M_{\infty}=0.85$, with angle of attack up to $5^{\circ}$, in the Göttingen transonic wind tunnel. A random dot pattern was applied on the surface, where the stereo camera pair was focused, and the wind off and wind on images (the latter with aerodynamic load) were compared for several camera viewing angles. They concluded that the camera position relative to the shock structure determined whether optical distortion due to aerodynamic effects was observed. They also pointed out that the range of aero-optically sensitive viewing angles is quite narrow, so density effects can be reduced by avoiding this area. Finally, they highlighted that a separation of the real (constant) and virtual model deformation (dependent on camera positioning) was enabled by the usage of multiple stereoscopic systems. Based on the work of Kirmse et al. (2010), however, it is not possible to assess whether pointtracking photogrammetry would be affected by optical distortion in the same way as IPCT.

Winter et al. (2018) investigated the impact of shock structures on optical distortion in a Mach 6 hypersonic flow. Two black anodised aluminum plates, equipped with laser-etched surface patterns, were mounted on each side of a $15^{\circ}$ wedge, with angle of attack varying between $0^{\circ}$ and $-15^{\circ}$. Imaging of the plate pattern was performed using different cameras and illumination strategies with frame rates between $3 \mathrm{~Hz}$ and $200 \mathrm{kHz}$. They quantified optical distortion using Strehl number ratios and only detected measurable optical distortion at the highest angle of attack $\left(-15^{\circ}\right)$, increasing with reservoir pressure. They also observed that tunnel vibrations at frequencies below $30 \mathrm{~Hz}$ significantly affected the measurements. However, from the work of Winter et al. (2018), it is not clear how optical distortion would affect the accuracy of a photogrammetric system.

Morris et al. (2018) performed experiments in the Arnold Engineering Development Test Propulsion Wind Tunnel 16T (at wind off conditions) with the Optical Model Attitude and Deformation System (OMADS, an optical measurement technique based on point-tracking photogrammetry using 185 fluorescent targets) and a high accuracy laser measurement device for method refinement and uncertainty quantification. The model, a $3 \times 0.6 \times 0.01 \mathrm{~m}$ aluminum plate, was installed in the wind tunnel and loaded with weights to generate deformations. Based on the difference between the OMADS and laser results, and using statistical methods, they observed that the camera configuration, defined in terms of viewing angle, target resolution, interior calibration, and 
camera hardware, had a clear effect on uncertainty. However, the experiments of Morris et al. (2018) were performed at wind off conditions; hence, it is not possible to comment on the effect of density gradients based on their work.

Schairer et al. (2017) employed stereo photogrammetry to measure position and attitude of a slender body of revolution in the NASA Ames $9 \times 7 \mathrm{ft}$ supersonic wind tunnel. Targets were placed on the body and on the wind tunnel walls for external orientation. They quantified calibration and random errors, but did not consider the effect of optical distortion.

The previous work by Kirmse et al. (2010), Winter et al. (2018), Morris et al. (2018), and Schairer et al. (2017) has highlighted that optical distortion affects the performance of optical techniques, and that the error introduced is a function of relative position of the cameras, surface of interest, and shock structures in the flow. However, the effect of density changes on the accuracy of point-tracking photogrammetry has not been adequately characterized. In this paper, we explore quantitatively the sources of optical distortion when applying point-tracking photogrammetry to experiments on a solid flat floor in the Imperial College supersonic wind tunnel. In addition, we present an application to a flexible panel in supersonic flow.

\section{Experimental setup}

Experiments were carried out in the Imperial College supersonic wind tunnel, a schematic of which is shown in Fig. 1. The tunnel is run in a blow-down mode of operation: high-pressure air is stored at $27 \mathrm{bar}$ in high-pressure tanks of total volume $48 \mathrm{~m}^{3}$, and is released to atmosphere. The maximum allowed run time is approximately $40 \mathrm{~s}$, including the start-up and shut-down periods. The working section has a constant square cross-sectional area with dimensions $150 \times 150 \times 727 \mathrm{~mm}$. The nozzle blocks can be changed to achieve two different supersonic Mach numbers in the working section ( $M=1.4$ and $M=2)$. A LabVIEW program, consisting of a proportional-integral-derivative controller, is used to operate the tunnel. It monitors the pressure in the settling chamber and adjusts the aperture of a pneumatic control valve to maintain the desired inlet stagnation pressure, which is typically achieved with a standard deviation of $0.16 \%$ (Gramola et al. 2018).

Figure 2 shows the experimental setup. Four synchronized high-speed cameras are placed on either side of the working section. Videos are recorded at $100 \mathrm{~Hz}$ to match the sampling frequency of the pressure transducers. Two models, placed on the wind tunnel floor, are used for the experiments described in this paper: a solid plate to characterize the photogrammetry technique, and a flexible plate to show an application to a deforming structure. Eighty 12-bit coded targets, of thickness $20 \mu \mathrm{m}$, are cut out of vinyl and attached to the model. Figure $3 \mathrm{a}$ shows a picture of the solid plate with the 80 coded targets, and Fig. $3 \mathrm{~b}$ shows the CAD sketch used to cut the vinyl using a CAMM-1 PRO Roland vinyl cutter.

\subsection{Camera calibration}

The commercial photogrammetry software package PhotoModeler Motion 2014.0.2 (Eos Systems Inc. 2014) is used for the camera calibration and target registration processes. For high accuracy, it requires that intrinsic and extrinsic camera calibrations are performed separately, and the constraints of the wind tunnel geometry mean that intrinsic camera calibration must be performed off site. Therefore, the cameras are initially placed around the wind tunnel, and focused in the middle of the surface of interest, with a depth of field sufficient to ensure that the targets are sufficiently sharp. All the camera and lens parameters are left the same for the whole duration of the project and they are listed in Table 1. It should be noted that some differences in aperture and exposure time between the cameras are due to the need

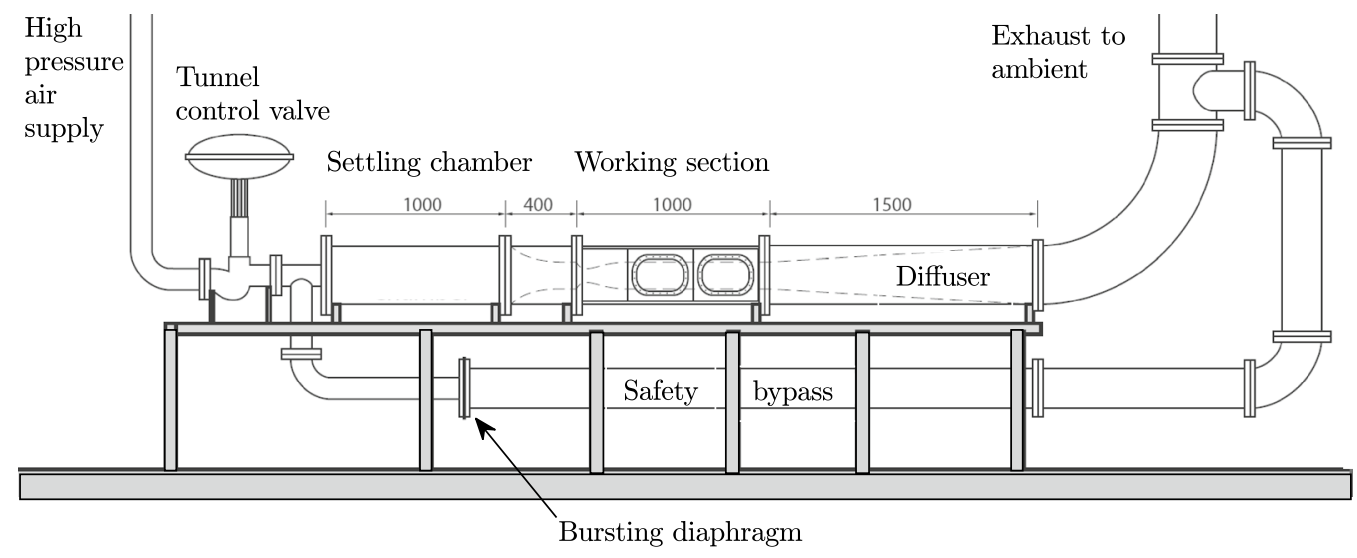

Fig. 1 Schematic of the Imperial College supersonic wind tunnel (Threadgill and Bruce 2017) 


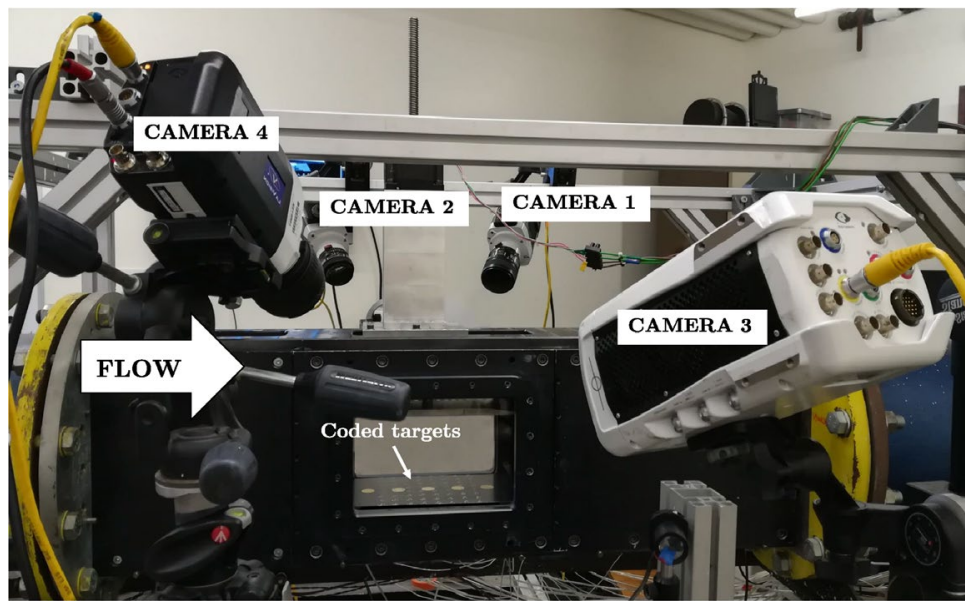

(a) Setup of the four high speed cameras around the Imperial College supersonic wind tunnel.

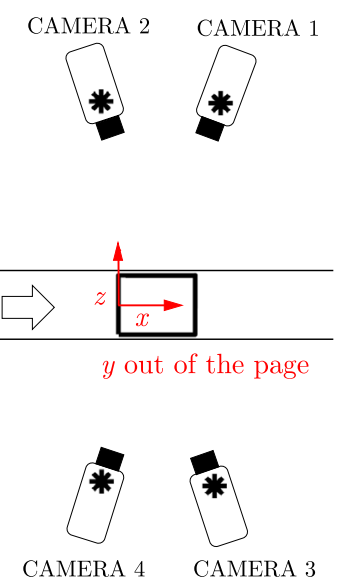

(b) Schematic showing the camera setup and the coordinate system (top view). The exact camera position is marked by a black asterisk.

Fig. 2 Experimental setup for the photogrammetry experiments

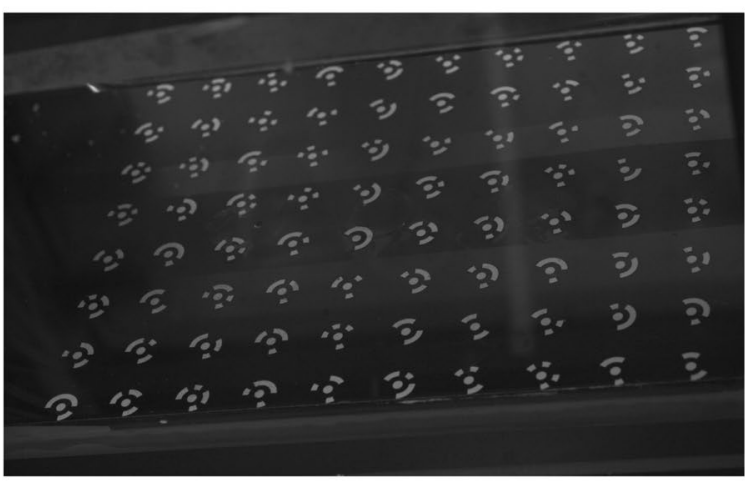

(a) Picture taken from camera 1.

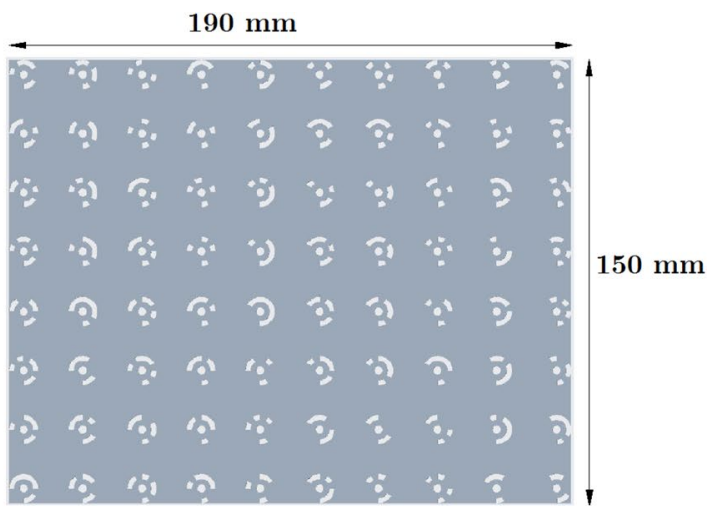

(b) Target arrangement, taken from CAD software.

Fig. 3 View of the model with the 80 coded targets

Table 1 Camera and lens parameters

\begin{tabular}{lllll}
\hline Label & 1 & 2 & 3 & 4 \\
\hline Camera type & Phantom Miro 310 & Phantom Miro 310 & Phantom v641 & Phantom Miro 310 \\
Lens & Nikon 60mm f2.8 D AF & Nikon 50mm f1.4 D AF & Nikon 50mm f1.8 D AF & Nikon 50mm f1.8 D AF \\
Aperture & 8 & 5.6 & 5.6 & 11 \\
Exposure time $(\mu \mathrm{s})$ & 9900 & 9900 & 9900 & 5000 \\
Image size (pixel) & $1280 \times 800$ & $1280 \times 800$ & $2560 \times 1600$ & $1280 \times 800$ \\
Frame rate $(\mathrm{Hz})$ & 100 & 100 & 100 & 100 \\
\hline
\end{tabular}

to maximize contrast in the images, given directionally nonuniform illumination in the laboratory.

The intrinsic camera parameters are the camera focal length, principal point and lens correction factors. Following
Zhang et al. (2010), intrinsic camera calibration is performed off site by taking several pictures of a known calibration grid, made up of a combination of dots and coded targets, laser printed on a flat sheet of paper. The size of the 
calibration grid matches the model to be tested in the wind tunnel, and the photos are taken at a distance similar to that between the cameras and the model. By processing these calibration pictures using PhotoModeler's Camera Calibrator module, the focal length, principal point, and lens distortion parameters (radial distortions $\mathrm{K}_{1}$ and $\mathrm{K}_{2}$, decentering distortions $\mathrm{P}_{1}$ and $\mathrm{P}_{2}$ ) are determined.

Extrinsic camera calibration, i.e., the determination of camera positions and orientations, is also performed automatically in every PhotoModeler project using a bundle adjustment algorithm (Eos Systems Inc. 2014).

\section{Results}

\subsection{Calibration and error estimation}

Experiments to explore the accuracy of the photogrammetry technique have been performed with a solid plate made from anodized aluminum placed on the wind tunnel floor, and the image distortion due to the glass windows and aerodynamic effects (e.g., shock waves and boundary layers) during a run is quantified. Data reduction is performed in PhotoModeler, which automatically identifies the corresponding features in different images with coded targets and determines 3D coordinates by triangulation. A bundle adjustment algorithm produces the best coordinates for the target points using an optimization process.

\subsubsection{Surface irregularities}

The 3D plate shape was reconstructed with the wind tunnel side windows removed. Figure 4 compares the plate height detected from photogrammetry (Fig. 4a) and using a Mazak VTC-200C II three-axis CNC machine equipped with a DTI gage, with overall accuracy of $3 \mu \mathrm{m}$ (Fig. 4b). The dots correspond to the detected positions of the coded targets, and a 3D surface was then fitted through them, using a thin-plate spline interpolation in MATLAB ('thinplateinterp'). In both cases, it can be seen that the plate appears almost flat, with small surface irregularities focused around the centerline, thought to be caused by black matt paint, which was sprayed to cover some shiny brass plugs. The standard deviation of the target height is $0.013 \mathrm{~mm}$ and $0.011 \mathrm{~mm}$ for Fig. $4 \mathrm{a}$ and $\mathrm{b}$, respectively, and the qualitative plate shapes agree. Some small differences are due to the targets moving slightly over the course of wind tunnel runs, which was observed by inspection. It should be noted that two targets were significantly damaged, and, therefore, their height was not measured with the Mazak machine, but is the result of a spatial average. This comparison neatly illustrates the extremely high level of accuracy that can be reached with photogrammetry, while at the same time highlighting a potential weakness of the technique if targets become damaged. However, in most practical wind tunnel applications, larger displacements (of the order of $1 \mathrm{~mm}$ ) are of interest. Difference plots are shown in the following sections to improve the clarity of the results.

\subsubsection{Optical distortion due to the glass windows}

Due to the difference in refractive index between the air and the optical glass, it is expected that optical distortion will be imparted by the presence of the wind tunnel windows, made from BK7 optical glass with thickness $19 \mathrm{~mm}$. Figure 5a shows a comparison between two frames taken from camera 1 with the wind tunnel windows on (cyan targets) and removed (red targets). The apparent shift of the targets perceived by the camera was measured to lie in the range 7-11 mm. Calculations applying Snell's Law of refraction

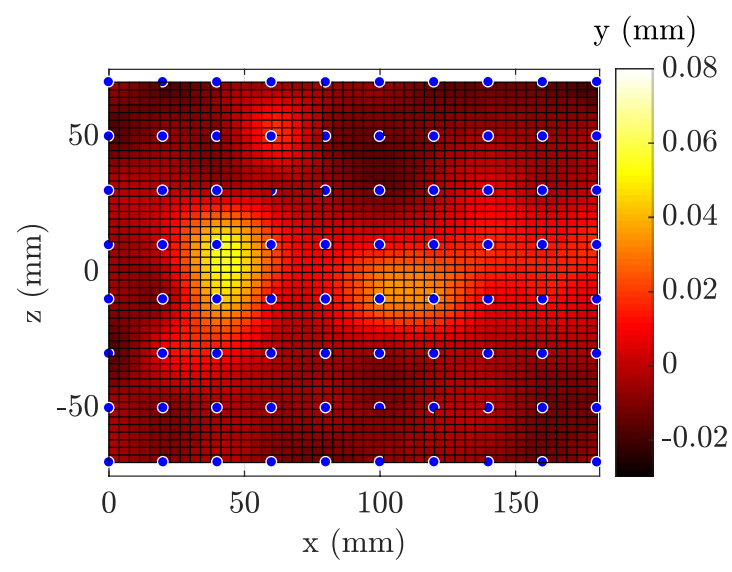

(a) From photogrammetry

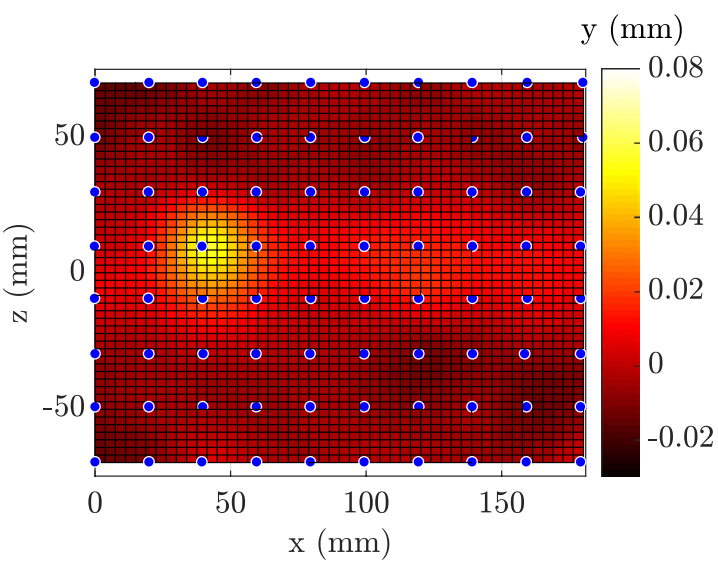

(b) From Mazak machine

Fig. 4 3D reconstruction of the wind tunnel floor piece with the side windows removed 


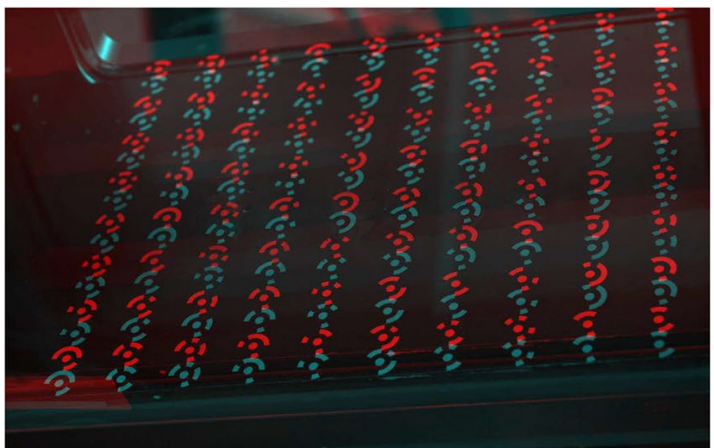

(a) No correction for the glass.

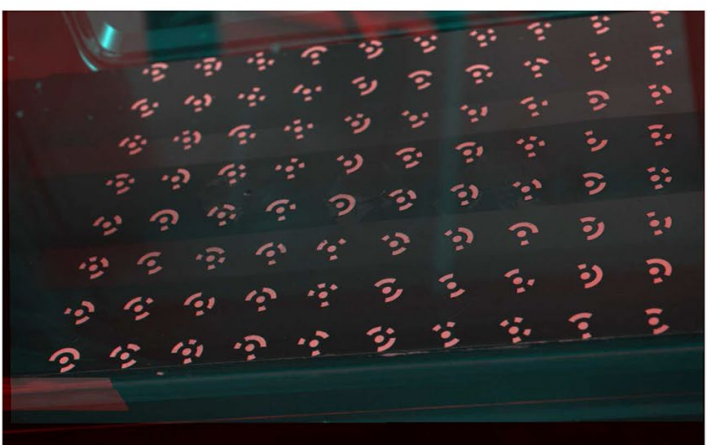

(b) Correction for the glass.

Fig. 5 Comparison of frames from camera 1 with the side windows on (cyan targets) and removed (red targets)

estimated this shift to be of the order of $10 \mathrm{~mm}$, in excellent agreement with the measured values. To understand the effect of the glass windows, affine transformations are performed in MATLAB, and the undistorted picture is recovered, as shown in Fig. 5b, where only one set of targets can be identified.

Given that the apparent shift of the targets due to aerodynamic effects, which will be described in Sect. 3.1.3, is significantly smaller than the distortion induced by the glass, it was found that correcting for the glass distortion in each image before processing it in PhotoModeler was introducing inaccuracies in the extrinsic calibration and target registration process. Therefore, it was decided to compare the target positions determined from PhotoModeler with the wind tunnel windows on and removed (at no flow conditions) for several picture combinations. Scaling factors were calculated to account for the apparent vertical displacement of each target due to the presence of the glass windows. These factors are the ratio of the target heights with the windows on and removed, averaged over the picture combinations considered. 2D spatial smoothing was subsequently applied to further improve accuracy.

\subsubsection{Optical distortion due to aerodynamic effects}

When the wind tunnel is running, the density of the air in the test section is lower than that of the air in the laboratory, shock waves, and supersonic boundary layers appear, and further distortion is, therefore, expected. To consider a wide range of flow conditions, calibration experiments are performed with an unconstrained normal shock at Mach 1.4, and with $8^{\circ}$ and $12^{\circ}$ oblique shocks at Mach 2 , as shown in Fig. 6 . The leading edge angle and height $(h)$ of the shock generator are changed to vary shock strength and impingement point for the runs with an oblique shock.

3D models of the plate were generated from the four synchronized videos. Correcting for the distortion caused by the glass and the initial surface unevenness due to

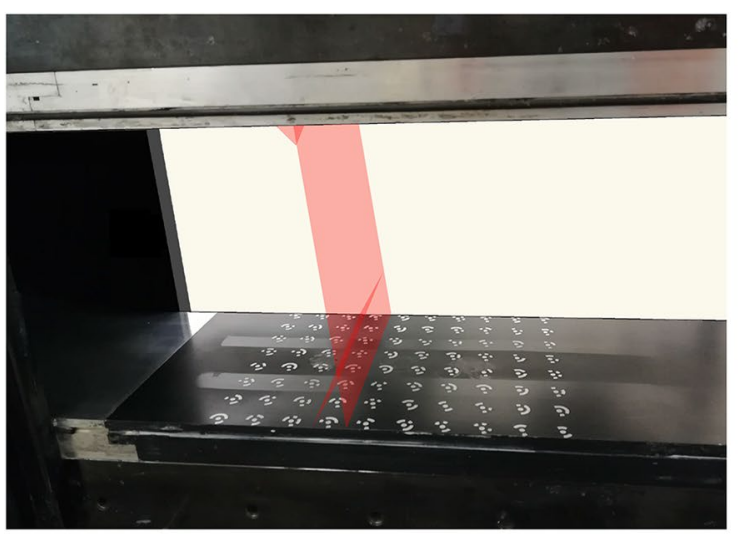

(a) Normal shock $(M=1.4)$.

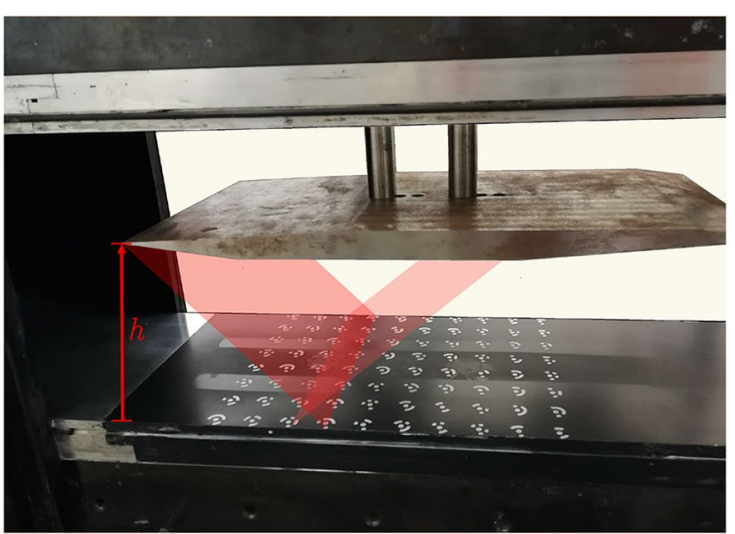

(b) Oblique shock $(M=2)$.

Fig. 6 Wind tunnel setup for the calibration experiments and shock structures expected 
the paint, the floor piece appeared flat, with very small differences in height across the surface (of the order of $0.01 \mathrm{~mm}$ ). Figure 7 shows the instantaneous RMS error $(\sigma)$ in plate height against normalized stagnation pressure in the tunnel throughout a number of tests with a $M=1.4$ normal shock and a $M=2$ oblique shock. The dotted vertical lines mark the appearance of the shock wave in the working section. The RMS error is defined as follows:

$\sigma=\sqrt{\frac{\sum_{i=1}^{N}\left(y(i)-y_{0}(i)\right)^{2}}{N-1}}$,

where $y(i)$ is the height of a target, $N$ the total number of targets, and the subscript 0 indicates the beginning of the experiment. The maximum RMS error was found to be 0.026 $\mathrm{mm}$, and this is attributed to optical distortion caused by aerodynamic effects, which can be further broken down into two contributions: (1) the lower pressure (and, therefore, density) in the working section, and (2) the presence of a compressible boundary layer, a shock wave, and the interaction between the two.

Considering the region of Fig. 7a and $\mathrm{b}$ to the left of the dotted lines, it can be seen that, without a shock wave in the working section, there is an almost linear relationship between optical distortion (represented by target height) and stagnation pressure ratio in the tunnel (with coefficient of determination $R^{2}=0.59$ and $R^{2}=0.43$ for the normal and oblique shock experiments, respectively). This is attributed to variations in density of the air in the test section during the wind tunnel start-up process, when a shock wave appears in the diverging part of the nozzle and travels downstream.

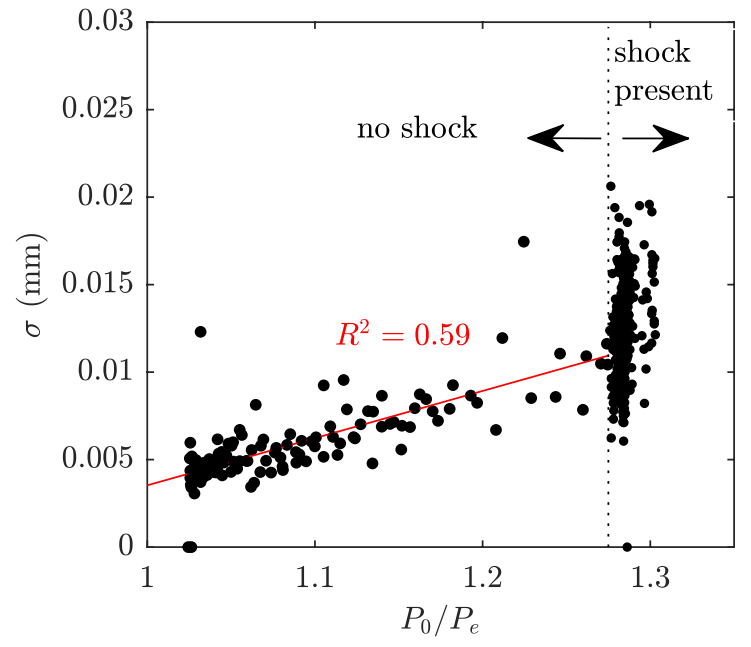

(a) Normal shock $(M=1.4)$
To explore this further, Fig. 8 shows the variation of detected height of a representative target $\left(y_{\mathrm{t}}\right)$ against time for one run with a $M=1.4$ normal shock and one run with a $M=2$ oblique shock $\left(12^{\circ} \mathrm{SG}, h=129.5 \mathrm{~mm}\right)$. Figure 8 indicates that the appearance of a shock wave is associated with a sudden rise in target height in both test cases. When the shock is in the working section (to the right of the dotted line in Fig. 8), the target heights oscillate around mean values. Comparing the two profiles in Fig. 8, it can be seen that this mean value is lower with a normal shock than with an oblique shock. This is believed to be due to a combination of differences in geometry and strength of the two shock wave/boundary layer interaction (SBLI) cases.

2D cross correlation of the images with and without flow from each of the four cameras, performed in MATLAB, has shown that the shock wave has imperceptible effect on the local optical distortion in individual frames. Furthermore, the reconstruction process to obtain the plate profile from the individual frames taken from different angles (as per Fig. 2) smooths out any optical distortion due to aerodynamic effects and increases accuracy. The RMS distortion for the individual frames of a representative run with a $M=2$ oblique shock $\left(12^{\circ} \mathrm{SG}, h=129.5 \mathrm{~mm}\right)$ at $t=15 \mathrm{~s}$ was found to be $28.4 \mu \mathrm{m}, 31.8 \mu \mathrm{m}, 25.9 \mu \mathrm{m}$, and $19.3 \mu \mathrm{m}$ (cameras 1, 2 , 3 , and 4 , respectively), while it dropped to $19.0 \mu \mathrm{m}$ for the final plate profile from photogrammetry. This explains why a discontinuity in target height is not seen around the shock wave location, a correlation was not found between shock position and optical distortion in the normal shock tests, and no clear difference was observed between the oblique shock runs with different shock impingement points.

Given that the flow structure above a flexible control device will inevitably be different from the flow above a

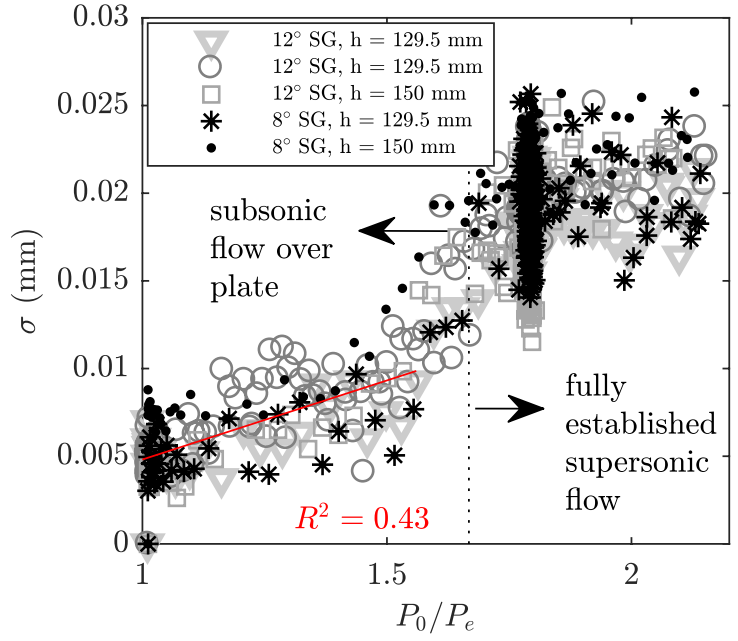

(b) Oblique shock $(M=2)$

Fig. 7 RMS error in plate height against normalized tunnel stagnation pressure 


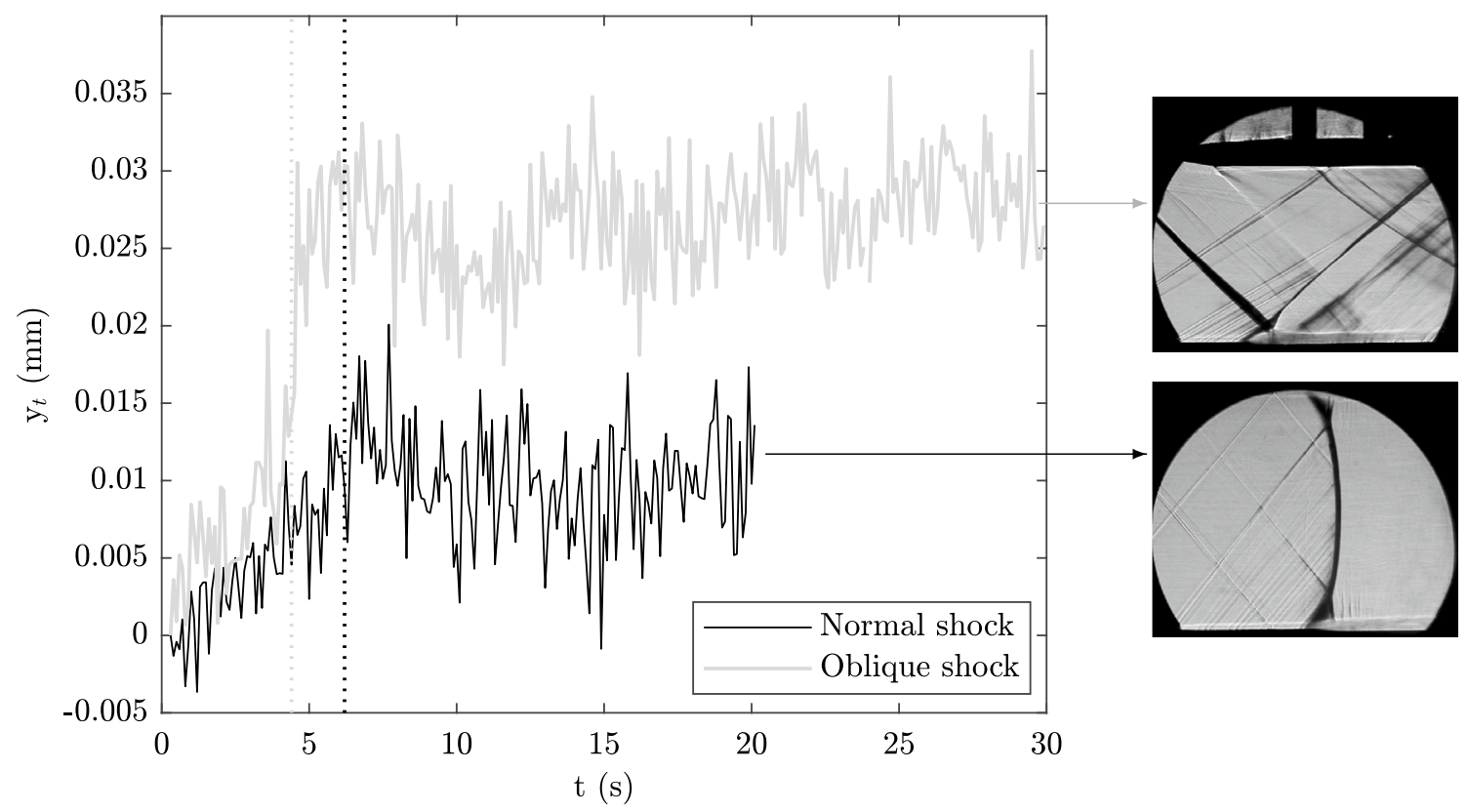

Fig. 8 Variation of detected height of a representative target $\left(y_{\mathrm{t}}\right)$ against time for one run with a $M=1.4$ normal shock and one with a $M=2$ oblique shock $\left(12^{\circ} \mathrm{SG} ; h=129.5 \mathrm{~mm}\right)$

solid plate, it is not possible to add a correction factor to account for the optical distortion caused by aerodynamic effects from the experimental data currently available. However, Fig. 7 shows that the error in the calculated displacement is expected to be small $(<0.03 \mathrm{~mm})$, and, therefore, negligible for applications with significant displacements (of the order of $1 \mathrm{~mm}$ ).

\subsubsection{Sources of uncertainty}

Two sources of uncertainty in this project are calibration and pixel uncertainty.

Calibration uncertainty As explained in Sect. 2.1, camera calibration is performed with PhotoModeler, and small uncertainties in the camera parameters could have affected the results. However, having used the same four cameras (and intrinsic parameters) for all the experiments presented, the errors introduced by these uncertainties are expected to be similar in all the frames, giving confidence to the accuracy of the relative results. Small changes in the location of the cameras between the experiments are accounted for by performing the extrinsic camera calibration before the target registration process for each project.

Pixel uncertainty An additional source of error in this project is the uncertainty in target position due to imperfect target location. PhotoModeler outputs residual values, to specify the disagreement between the marked locations of the targets on each photograph and the predicted location, based on where the projection of the $3 \mathrm{D}$ points falls on the photo. The residuals were found to be of the order of 0.1 pixel (where 1 pixel $\approx 0.15 \mathrm{~mm}$ ) for all the targets. In addition, the processing algorithm calculates precision values in the $x$-, $y$-, and $z$-directions, to specify how precise the locations of the $3 \mathrm{D}$ points are in the $3 \mathrm{D}$ space. The precision in the vertical $(y)$ direction is of the order of $0.01 \mathrm{~mm}$, and was found to be highly dependent on target location. Given this dependence, and the fact that the target heights, e.g., the profiles shown in Fig. 8, show some clear trends, we infer that the pixel uncertainty is primarily a function of relative camera and target position, and, in the same way as the calibration uncertainty, has affected the different frames in a similar way, giving confidence to the relative results presented in this paper. The fact that the pixel uncertainty is of the same order of magnitude of the optical distortion due to aerodynamic effects, presented in Sect. 3.1.3, further indicates that, for the experimental setup tested, optical distortion can be considered negligible as it lies within the bounds of PhotoModeler's precision.

\subsection{Application to adaptive shock control bumps}

Photogrammetry has been applied to experiments on adaptive shock control bumps placed beneath a Mach 1.4 transonic shock wave, similar to those described by Gramola et al. (2018). The bump consists of an aluminum alloy plate with dimensions $308 \times 150 \times 0.6 \mathrm{~mm}$, glued to solid blocks, such that the flexible portion is a square with side $L=150 \mathrm{~mm}$. To minimize reflection, the plate was sprayed 
with matt black paint before the white vinyl targets were applied. Figure 9 shows the 3D reconstruction of the flexible bump, with the 80 dots marking the positions of the coded targets. From Fig. 9, it can be seen that the flexible plate exhibits considerable 3D deformation, particularly at the leading and trailing edges, due to the way that the plate is clamped to the wind tunnel floor.

The spanwise-averaged plate shape is also detected from shadowgraph (considering the bottom surface where density gradients in the air are negligible), and this is compared with several 2D profiles at different spanwise positions from photogrammetry in Fig. 10. Good agreement is observed with the lowest points among all the $2 \mathrm{D}$ profiles, which is consistent with how the plate shape was detected from shadowgraph. In addition, the 2D slices from photogrammetry show that the curvature at the leading edge of the flexible plate is not uniform in the spanwise direction (as it would be assumed from the 2D schlieren reconstruction), but it decreases from the centerline towards the wind tunnel side walls, with a variation in maximum slope of $25 \%$ across the span. For an upstream Mach number of 1.4 and a change in plate angle between $3^{\circ}$ and $4^{\circ}$, a spanwise decay of approximately $1.5^{\circ}(3 \%)$ is expected in the angle of the curvatureinduced oblique shock (Anderson 1990), revealing the existence of a curved front shock leg. This can be observed in the shadowgraph picture (Fig. 9), where the oblique shock

Fig. 9 3D reconstruction of an adaptive shock control bump from photogrammetry with the corresponding shadowgraph picture showing the $\lambda$-shock structure (dimensions in $\mathrm{mm}$ )
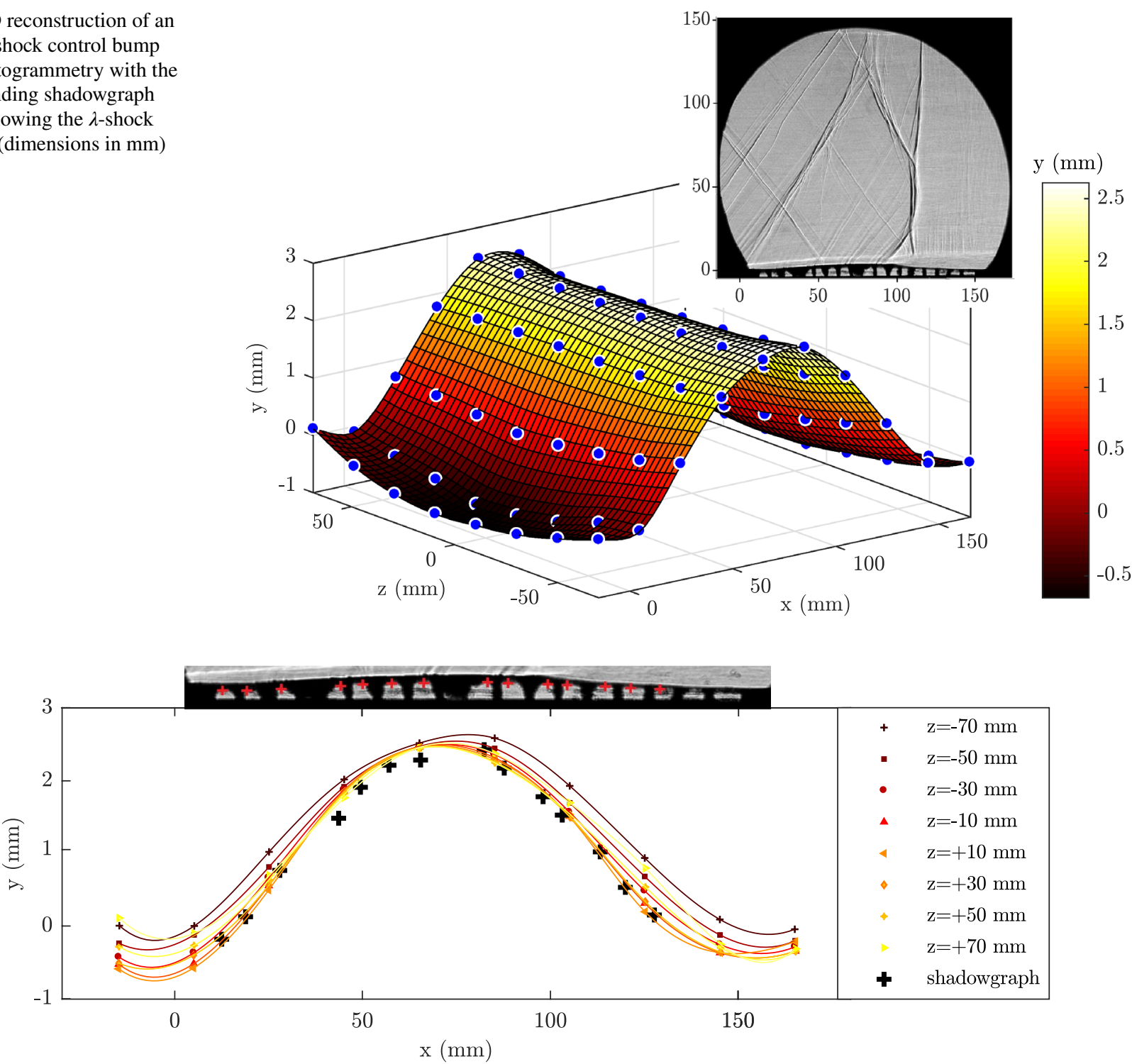

Fig. 10 2D plate profiles at different spanwise positions from photogrammetry, compared with the spanwise-averaged plate shape detected from shadowgraph (black crosses). A close-up view of the shadowgraph picture (aligned with the profiles) is included, and the detected points are marked as red crosses. The vertical segments at the bottom of the shadowgraph image are static pressure tappings 
is particularly thick close to the flexible plate, resembling schlieren images of shock structures above 3D shock control bumps (Bruce and Colliss 2015).

It can be concluded that the shape of the adaptive bump can be detected accurately from photogrammetry, including complex 3D effects that would not be picked up from spanwise-averaged techniques such as schlieren or shadowgraph.

\section{Conclusions}

It has been shown that point-tracking photogrammetry applied to supersonic wind tunnel experiments can reach high levels of accuracy, allowing reliable measurement of model deformations (with a root-mean-square error of the order of $0.01 \mathrm{~mm}$ and below $0.03 \mathrm{~mm}$ for all of the configurations tested here). The optical distortion associated with photogrammetry has been broken down into two contributions: the glass windows and aerodynamic effects, due to a change of pressure in the working section and the presence of shock waves. For the experimental setup tested, aerooptical distortion was found to increase with shock strength (between $M=1.4$ and $M=2$ ) and be insensitive to shock position, but to lie within the bounds of PhotoModeler's precision. Photogrammetry has been successfully applied to determine the 3D shape of an adaptive shock control bump beneath a $M=1.4$ transonic shock wave, revealing 3D deformations that could not have been detected with schlieren photography. This highlights the limitations of applying a 2D spanwise technique to accurately represent real surfaces.

Acknowledgements The first author wishes to acknowledge the Imperial College President's PhD Scholarship Scheme, supported by EPSRC, for their support of this research.

Open Access This article is distributed under the terms of the Creative Commons Attribution 4.0 International License (http://creativeco mmons.org/licenses/by/4.0/), which permits unrestricted use, distribution, and reproduction in any medium, provided you give appropriate credit to the original author(s) and the source, provide a link to the Creative Commons license, and indicate if changes were made.

\section{References}

Anderson JD (1990) Modern compressible flow: with historical perspective, vol 12. McGraw-Hill, New York

Baqersad J, Poozesh P, Niezrecki C, Avitabile P (2017) Photogrammetry and optical methods in structural dynamics-a review. Mech Syst Signal Process 86:17-34

Barrows D (2007) Videogrammetric model deformation measurement technique for wind tunnel applications. In: 45th AIAA aerospace sciences meeting and exhibit, $\mathrm{p} 1163$
Bruce PJK, Colliss SP (2015) Review of research into shock control bumps. Shock Waves 25(5):451-471

Burner AW, Lokos WA, Barrows DA (2003) In-flight aeroelastic measurement technique development. In: Optical diagnostics for fluids, solids, and combustion II, vol 5191, pp 186-200. International Society for Optics and Photonics

Castellini P, Chiariotti P, Martarelli M, Zappa E, Lavatelli A (2017) Experimental modal analysis on vibration data measured by digital image correlation. In: Shock and vibration, aircraft/aerospace, energy harvesting, acoustics and optics, vol 9, pp 285-291

Eos Systems Inc. (2014) Photomodeler user guide

Gramola M, Bruce PJK, Santer M (2018) Experimental FSI study of adaptive shock control bumps. J Fluids Struct 81:361-377

Graves SS, Burner AW, Edwards JW, Schuster DM (2003) Dynamic deformation measurements of an aeroelastic semispan model. J Aircraft 40(5):977-984

Grossman IJ, Bruce PJK (2018) Confinement effects on regular-irregular transition in shock-wave-boundary-layer interactions. J Fluid Mech 853:171-204

Kirmse T, Ebel PB, Schroeder A (2016) Image base fan blade deformation measurements on an Airbus A320 V2500 engine in ground operation. In: 54th AIAA aerospace sciences meeting, p 0386

Kirmse T, Gardner A, Krombholz C (2010) Density effects in model deformation measurements on a NACA0010 airfoil in a transonic flow. In: 14th international symposium on flow visualization

Morris C, Nelson M, Rought R (2018) Uncertainty analysis of the optical model attitude and deformation system in the AEDC PWT 16T facility. In: 2018 aerodynamic measurement technology and ground testing conference, $\mathrm{p} 4049$

Quix H, Semmelmann J, Wright M (2015) Model deformation measurement capabilities at ETW. In: 31st AIAA aerodynamic measurement technology and ground testing conference, p 2562

Schairer ET, Kushner LK, Drain BA, Heineck JT, Durston D (2017) Stereo photogrammetry measurements of the position and attitude of a nozzle-plume/shock-wave interaction model in the NASA Ames 9-by 7-ft supersonic wind tunnel. In: 55th AIAA aerospace sciences meeting, $\mathrm{p} 1053$

Schairer ET, Kushner LK, Heineck JT, Solis E (2018) Measurements of parachute dynamics in the worlds largest wind tunnel by stereo photogrammetry. In: 2018 aerodynamic measurement technology and ground testing Conference, $\mathrm{p} 3802$

Spain C, Heeg J, Ivanco T, Barrows D, Florance J, Burner A, DeMoss J, Lively P (2004) Assessing videogrammetry for static aeroelastic testing of a wind-tunnel model. In: 45th AIAA/ASME/ASCE/ AHS/ASC structures, structural dynamics and materials conference, p 1677

Threadgill JAS, Bruce PJK (2017) Comparison of unsteady flow similarities in various shock/boundary-layer interaction configurations. In: 55th AIAA aerospace sciences meeting, p 0986

Wang W, Li X, Ahmat Y, Hu X, Chen A (2018) Vibration measurement method based on point tracking for irregular structures. Optik

Winter M, Green R, Borchetta C, Josyula E, Hayes JR, Jewell JS, Hagen B (2018) Experimental investigation of image distortion in a Mach 6 hypersonic flow. In: 2018 aerodynamic measurement technology and ground testing conference, p 4197

Zhang ZY, Wang SL, Sun Y (2012) Videogrammetric measurement for model displacement in wind tunnel test. Appl Mech Mater 130:103-107 (Trans Tech Publ)

Zhang W, Jiang T, Han M (2010) Digital camera calibration method based on PhotoModeler. In: 2010 3rd international congress on image and signal processing (CISP), vol 3, pp 1235-1238. IEEE 


\section{Affiliations}

\section{Michela Gramola $^{1}$ (D) Paul J. K. Bruce ${ }^{1} \cdot$ Matthew Santer $^{1}$}

$\triangle$ Michela Gramola

michela.gramola14@imperial.ac.uk

Paul J. K. Bruce

p.bruce@imperial.ac.uk

Matthew Santer

m.santer@imperial.ac.uk
1 Department of Aeronautics, Imperial College London, London SW7 2AZ, UK 